\title{
A Modified Version of the CAGE as an Indicator of Alcohol Abuse and Its Consequences Among Undergraduate Drinkers
}

\author{
Carol J. Boyd, PhD, RN, FAAN, ${ }^{1,2}$ Sean Esteban McCabe, MSW, PhD, ${ }^{1}$ \\ and Hannah d'Arcy, $\mathrm{MS}^{1}$
}

In March of 1999, we conducted a cross-sectional, web-based survey using a random sample of 2041 undergraduate students attending a large, public university in the Midwest. The sample was recruited via e-mail and students completed the Web survey from their computer terminals. Toward the goal of establishing the usefulness of the $C A G E$ when screening undergraduates for alcohol misuse, we first examined the proportion of undergraduates with a positive score on a modified version of the CAGE and then determined the correlates of a positive CAGE score (e.g. alcohol consumption, fear of addiction, etc.). We found preliminary evidence that the question "Have you been afraid you might be alcoholic" may be the best identifier, when compared to the modified CAGE, of at-risk students.

KEY WORDS: CAGE; binge drinking; college students; alcohol use.

Heavy drinking on U.S. college campuses is a common phenomenon that poses a health threat to undergraduate college students. Indeed, for over 20 years, these students have had heavy episodic drinking rates that are far higher than their sameage peers not attending college and all other age groups (1). Approximately $44 \%$ of undergraduate students nationwide engaged in heavy episodic drinking at least once within the previous two weeks, compared to $37 \%$ who drank occasionally and $19 \%$ who were abstinent in the past year (2).

As universities and colleges grapple with the mental and physical health consequences of their students' heavy drinking, professionals ponder the best way to identify and assist these relatively young problem drinkers. Although a variety of short, alcohol-screening instruments for identifying problem drinkers are available, these screening instruments are not well studied in college populations. The few validity studies that have used college-based populations revealed that these instruments

\footnotetext{
${ }^{1}$ Substance Abuse Research Center, University of Michigan, Michigan.

${ }^{2}$ To whom correspondence should be addressed to Substance Abuse Research Center, University of Michigan, 475 Market Place, Suite D, Ann Arbor, Michigan 48108-1649; e-mail: caroboyd@umich.edu.
} 
often perform erratically; a major concern since reliable, valid, and practical screening instruments are critical for obtaining accurate assessments of problem drinking among college students (3-5).

Toward the goal of establishing the usefulness of the CAGE in assessing undergraduate alcohol abuse, this study aimed to answer the following questions:

What is the proportion of undergraduates that have a positive score on the CAGE?

Is there an association between CAGE scores and gender, race, and years in college?

Is there a relationship between the CAGE and the negative consequences of alcohol abuse?

In addition to these questions we explored two possible alternatives to the CAGE, examining their associations with other alcohol-use measures and with consequences of drinking.

\section{BACKGROUND}

Many universities and colleges use the CAGE to assess potential drinking problems among their students. Myerholtz and Rosenberg (6) conducted a survey of 100 randomly selected directors of collegiate alcohol programs. They found that directors most often reported using one of the following brief screening instruments when trying to identify problem drinkers among undergraduates: the Michigan Alcoholism Screening Test, the Substance Abuse Subtle Screening Inventory-2, the CAGE, or the MacAndrew Scale of the MMPI. One of these instruments, the CAGE (with only four items) is often viewed as the most practical because of its brevity and ease in administration $(7,8)$. Although there are relatively few studies that address the use of the CAGE with younger and/or nonclinical populations, there are concerns about the sensitivity and specificity of the CAGE, particularly when used with a nonclinical population (9) and with younger, university populations (4).

O'Hare and Tran (5) conducted a large student alcohol and drug survey at Rutgers University where 606 out of 800 randomly selected undergraduates responded to an anonymous, self-reported, mailed questionnaire. Students who reported drinking at least once in the past 12 months and also reported at least one drinking-related problem during the past year were included in the study. Assuming that a standard drink approximates $0.6 \mathrm{oz}$, O'Hare and Tran used a quantity and frequency measure that allowed them to develop a typology of absolute alcohol consumption: light (0.01-0.21 oz), moderate (0.22-0.99 oz), and heavy drinking (1.0 oz. and above). In addition, the authors used the CAGE questionnaire and an 18-item consequent inventory that was gleaned from other college surveys (5). Overall, O'Hare and Tran noted that the CAGE was not a good predictor of drinking-related problems in college students although like Cherpitel $(10,11)$, they found it was a better predictor for men. It appeared that the CAGE did not address drinking problems deemed relevant by college students despite these students' reports of negative consequences from their alcohol abuse. Indeed, O'Hare and Tran noted that a substantial number 
of students were concerned about their own drinking; however, the CAGE failed to reflect their concerns. It appears that despite the CAGE's popularity, a more specific measure for assessing alcohol problems, a measure that is both brief and easy to score, may be needed for collegiate populations.

\section{METHODS}

\section{Sample}

The undergraduate sample $(N=2041)$ was representative of the entire undergraduate student population at the University of Michigan with respect to gender, ethnicity, and membership in Greek social organizations. The sample included $49 \%$ females and 51\% males with the following ethnic distribution: White/Caucasian (72\%), Asian (12\%), African American (5\%), Hispanic (4\%), and other (7\%). Nineteen percent of respondents were members of a social fraternity or sorority organization.

Of the 2041 undergraduate respondents, 196 (9.6\%) had not used alcohol in the past year, and another $174(8.5 \%)$ had consumed alcohol in the past year but classified themselves as currently abstaining. In addition, 22 students could not be classified as drinkers or abstainers because they refused questions or gave "don't know" responses. The remaining 1649 undergraduate drinkers from the sample were used in all subsequent analyses.

\section{Design and Procedures}

In the winter semester of 1999, the student population at the University of Michigan in Ann Arbor included 35,061 students: 22,619 undergraduate and 12,442 graduate students; who together represented the student population sampled. After receiving approval from the University's IRB in the spring of 1999, the university registrar's office provided the authors with a list of 4000 randomly selected undergraduate and graduate students; it is the undergraduate portion of the sample that we examine here. Students were told that if they completed the survey, they would be eligible for a draw that included such prizes as free airline tickets, football tickets, t-shirts, and coupons to local businesses. Our final response rate was 68\%.

In the Student Life Survey we asked students to divulge information about sensitive and illegal behaviors. We assumed that students would be reluctant to answer questions if they felt that the University-or faculty related to the Universityhad access to their individual responses. Therefore, we engaged in several strategies to protect the respondent's confidentiality. We contracted with a research firm that was unaffiliated with the university. This firm assisted us with our Web site as well as stored and maintained the student data files. University officials, faculty and staff were unable to access the names, e-mail addresses or data of any individual respondent, and respondents were told this when they received the first e-mail. The data file containing the respondent's identifying information was stored in a password-protected location at the independent research firm, while name and 
responses were disengaged and could not be linked. The survey was maintained on a hosted secure Internet site running under the secure socket layer (SSL) protocol, and the respondent's data could not be matched to the respondent's identifying user ID.

\section{Questionnaire}

The Student Life Survey (SLS) was originally developed and pilot tested in 1993 using a paper and pencil format. The SLS draws from items in the Monitoring the Future study (1), CORE survey (12), and the College Alcohol Study (13). The webbased survey took approximately $20 \mathrm{~min}$ to complete. Study design and procedures for the SLS are described in more detail elsewhere (14).

Students completed a demographic section of the questionnaire including questions about, among other items, age, sex, living arrangement, grade point average, and year in school. Students were given a slightly modified version of the CAGE questionnaire and also asked a series of questions about their drinking behaviors and attitudes (e.g. how much alcohol they usually consume, where they usually drink, motivations for drinking, and perceived consequences of drinking). And finally, although we are not reporting the data here, students were asked detailed questions about their illegal and legal substance use, local policies related to drug use, and their perceptions of their friends' drug use.

\section{Measurement}

Students who classified themselves as abstainers from alcohol were not given the CAGE questions because of a skip pattern embedded in the Web questionnaire. Therefore, these students are excluded from our analyses. Students who used alcohol were asked how many times in the past year they had experienced each of the four CAGE criteria: C: "Felt that you should cut down your drinking"; A: "Been annoyed by people criticizing your drinking"; G: "Felt guilt or remorse after drinking," and E: "Had a drink first thing in the morning as an 'eye opener.'" If students indicated that they had experienced two or more of these events in the past year, this was considered a "positive" screening test result, denoting suspected alcohol abuse. If students had experienced none or only one of the four criteria, they were assigned a "negative" test result. This is standard scoring for the CAGE instrument (15).

In addition to the modified CAGE items, the survey contained several negative consequences of alcohol use in the past year that were adapted directly from the College Alcohol Study (e.g. had a hangover, missed a class, got behind in school work, done something you later regretted, had a memory loss, argued with friends, engaged in unplanned sex, damaged property, got in trouble with campus or local police, got hurt or injured, drove under the influence of alcohol, arrested for driving under the influence). The survey also contained some alcohol-related behaviors that we were interested in testing as possible items to improve screening instruments for alcohol abuse and alcoholism (e.g. vomited, were afraid you might be alcoholic, thought about suicide). 


\section{ANALYSES AND DISCUSSION}

The purpose of the present study was to evaluate the usefulness of the CAGE in assessing undergraduate alcohol abuse. In order to determine the usefulness of the CAGE we first determined the proportion of undergraduates who have a positive score on the CAGE and then examined how this proportion varied by gender, race, class, and year. In addition, we examined the relationship between the CAGE and other correlates of alcohol consumption, including the negative consequences associated with heavy drinking. Secondarily, we explored two possible alternatives to the CAGE, examining these alternatives in relation to consequences of drinking.

Overall, the CAGE instrument flagged $22.7 \%$ of the sample as probable alcohol abusers. Over a quarter of men $(26.0 \%)$ had positive CAGE results, compared with $19.5 \%$ of women, a significant difference $(p=0.002)$. Underclassmen, and in particular freshmen, appeared more likely to be flagged as potential alcohol abusers. Ethnicity was only marginally associated with CAGE results, with Asian and African American students least likely to test positive. Students who were members of a fraternity or sorority were significantly more likely than nonmembers to test positive, but whether members lived in a fraternity or sorority house or lived elsewhere was not apparently associated with the likelihood of a positive CAGE result (See Table I).

Respondents reported the number of times they used alcohol in the past year, and the number of times they had gotten drunk in the same time period. We calculated the proportion of students in each response category who were flagged as problematic

Table I. Key Characteristics of Undergraduate Drinkers, and Percentage Testing Positive on the CAGE Within Each Subgroup

\begin{tabular}{lccc}
\hline \multicolumn{1}{c}{ Student characteristic } & Overall $n(\%)$ & \% Positive on CAGE & Chi-square $p$-value \\
\hline Overall & $1649(100)$ & 22.7 & - \\
Gender & & & 0.002 \\
Male & $805(48.8)$ & 26.0 & \\
Female & $844(51.2)$ & 19.5 & 0.055 \\
Race & $1188(72.0)$ & 23.2 & \\
White & $142(8.6)$ & 16.4 & \\
Asian & $54(3.3)$ & 13.0 & \\
African American & $265(16.1)$ & 25.8 & \\
Other/refused & $373(22.6)$ & & \\
Class year & $401(24.3)$ & 27.9 & \\
Freshman & $407(24.7)$ & 22.7 & \\
Sophomore & $468(28.4)$ & 19.8 & \\
Junior & $1332(81.2)$ & 21.0 & \\
Senior & $177(10.8)$ & 21.2 & \\
Fraternity/Sorority & $132(8.0)$ & 29.1 & \\
Nonmember & $372(22.7)$ & 28.1 & \\
Member & $430(26.3)$ & 17.3 & \\
Live-in member & $458(28.0)$ & 17.4 & \\
Alcohol use (past 12 months) & $378(23.1)$ & 21.0 & \\
$\quad$ None & & 36.3 & \\
Less than monthly & & & \\
Monthly & & & \\
Weekly to daily & & & \\
\hline
\end{tabular}


Table II. Self-reported Alcohol Use and its Association With the CAGE

\begin{tabular}{lccc}
\hline \multicolumn{1}{c}{ Self-reported alcohol use } & $\begin{array}{c}\text { Overall } \\
n(\%)\end{array}$ & $\begin{array}{r}\text { \% Positive } \\
\text { on CAGE }\end{array}$ & $\begin{array}{c}\text { Chi-square } \\
p \text {-value }\end{array}$ \\
\hline \# Times consumed alcohol in past year & & & \\
$1-2$ & $44(2.8)$ & 0.3 & $<0.001$ \\
$3-5$ & $129(8.1)$ & 2.5 & \\
$6-9$ & $154(9.7)$ & 5.0 & \\
$10-19$ & $305(19.2)$ & 12.8 & \\
$20-39$ & $368(23.1)$ & 20.6 & \\
40 to more & $592(37.2)$ & 58.9 & \\
\# Times got drunk in past year & & & \\
None & $86(5.9)$ & 1.4 & $<0.001$ \\
1-2 & $319(21.9)$ & 7.7 & \\
3-5 & $258(17.7)$ & 15.7 & \\
6-9 & $188(12.9)$ & 13.1 & \\
10-19 & $208(14.3)$ & 17.7 & \\
20-39 & $193(13.2)$ & 17.1 & \\
40 to more & $206(14.1)$ & 27.1 & \\
Self-description of alcohol use & & & \\
Occasional drinker & $751(45.9)$ & 29.4 & $<0.001$ \\
Light drinker & $307(18.8)$ & 11.5 & \\
Moderate drinker & $502(30.7)$ & 46.4 & \\
Heavy drinker & $75(4.6)$ & 12.6 & \\
\hline
\end{tabular}

drinkers based on the CAGE scores. While the percentage testing positive on the CAGE increased as the frequency of drinking increased, we did note some anomalies. Of those who reported using alcohol at least 40 times in the past year (592 students), $58.9 \%$ were identified as probable alcohol abusers. However, of those who reported getting drunk at least 40 times (206 students) the percentage flagged by the CAGE was only $27.1 \%$.

A subjective measure of drinking behavior was also available. Respondents were asked whether they considered themselves occasional, light, moderate, or heavy drinkers (abstainers are excluded here). The CAGE was not correlated with this selfclassification variable in any consistent manner (See Table II). This discordance may by explained by the fact that "occasional" is an assessment of frequency whereas "light," "moderate," and "heavy" are indicators of quantity.

An important goal of collegiate programs aimed at preventing heavy alcohol use is to diminish the negative consequences that can result from such use. With this in mind, we examined the association between the CAGE scores and the prevalence of 18 distinct and negative consequences. In all 18 cases, students with positive CAGE results were more likely to experience the given consequence. The strongest association was seen with the item "Were afraid you might be alcoholic"; in fact, students were over 17 times more likely to have experienced this fear if their CAGE results were positive rather than negative. Most often, a positive CAGE score was associated with having three to five times the odds of experiencing a given consequence.

We considered whether association with the CAGE scores varied depending on gender for any of the consequences; however, we did not find that the odds ratios for men and women differed in a statistically significant manner (See Table III). The results did suggest that among women, the CAGE was more highly associated with the items "Someone said you should cut down" and "Were afraid you might be 
Table III. Experience of Negative Consequences of Alcohol Use in the Past Year

\begin{tabular}{lcccr}
\hline & \multicolumn{2}{c}{ \% Experiencing consequence } & \\
\cline { 2 - 4 } \multicolumn{1}{c}{ Consequence } & Overall & CAGE + & CAGE - & OR \\
\hline Had hangover & 77 & 92 & 72 & 4.5 \\
Vomited & 68 & 88 & 62 & 4.6 \\
Felt embarrassed & 56 & 84 & 47 & 5.7 \\
Had memory loss & 41 & 70 & 33 & 4.8 \\
Missed class & 39 & 61 & 32 & 3.2 \\
Drove drunk & 30 & 45 & 26 & 2.4 \\
Got into an argument or fight & 24 & 44 & 18 & 3.7 \\
Were hurt or injured & 17 & 36 & 11 & 4.4 \\
Someone said to cut down & 12 & 38 & 5 & 12.2 \\
Performed poorly on a test & 11 & 26 & 7 & 4.6 \\
Were sexually harassed & 11 & 22 & 8 & 3.4 \\
Damaged property & 9 & 18 & 6 & 3.4 \\
Had trouble with police & 9 & 17 & 6 & 3.1 \\
Were afraid might be alcoholic & 7 & 24 & 2 & 17.1 \\
Sexually harassed someone & 4 & 9 & 3 & 3.0 \\
Friend threatened to leave & 4 & 9 & 2 & 4.2 \\
Thought about suicide & 3 & 10 & 1 & 7.2 \\
Were arrested for DUI & 1 & 1.9 & 0.2 & 8.1 \\
\hline Note. Percentages & &
\end{tabular}

Note. Percentages are given for overall and for subgroups testing positive and negative on the CAGE. Odds ratios represent the greater odds of each consequence associated with a positive CAGE result. In all cases, odds ratios are significant, $p<0.05$.

alcoholic," but these trends were only marginally significant $(p<0.10)$. For both men and women, "Were afraid you might be alcoholic" showed the greatest correlation with CAGE results. Women testing positive on the CAGE were over 30 times more likely to fear they were alcoholic, while for men the odds were about 12 times greater.

Because students in fraternity and sorority social organizations have different drinking behaviors and attitudes $(12,16,17)$ from those who are not affiliated, it was plausible that the CAGE might be more useful in one group than the other. We explored this possibility but found no statistically significant differences between members and nonmembers in the degree of association between CAGE and any of the consequences. Once again, a fear that one might be alcoholic was most strongly correlated with the CAGE, both for members and nonmembers.

While the CAGE shows reasonable association with frequency of drinking and with the negative consequences of drinking, we also explored two alternatives for identifying probable alcohol abusers in this population. First, we selected the "Were afraid you might be alcoholic" item, because of its simplicity (a single question) and its correlation with the CAGE. Secondly, we considered any student who said they were a moderate or heavy drinker as potentially at risk. Both alternatives thus depend on the student's self-assessment of drinking behavior.

In Table IV, we give the percentage of students who tested positive for each of our alternative criteria, and we repeat the percentage testing positive on the CAGE for ease of comparison. (Note: The prevalence for each is given overall and within various drinking behavior categories, as in Table II.) Each alternative measure showed a stronger association than the CAGE with the frequency of alcohol consumption in the past year. While only $7.0 \%$ of the total sample reported being afraid they were 
Table IV. Comparison of Three Potential Indicators of Problematic Alcohol Use, and Their Relation to Self-reported Alcohol Consumption

\begin{tabular}{|c|c|c|c|c|}
\hline Self-reported alcohol use & $\begin{array}{l}\text { Overall } \\
n(\%)\end{array}$ & $\begin{array}{l}\% \text { Afraid } \\
\text { alcoholic }\end{array}$ & $\begin{array}{l}\% \text { Moderate or } \\
\text { heavy drinkers }\end{array}$ & $\begin{array}{l}\% \text { Positive } \\
\text { on CAGE }\end{array}$ \\
\hline Overall & $1649(100)$ & 7.0 & 35.3 & 22.7 \\
\hline \multicolumn{5}{|l|}{$\begin{array}{l}\text { \# Times consumed alcohol } \\
\text { in past year }\end{array}$} \\
\hline $1-2$ & $44(2.8)$ & 0.0 & 0.0 & 0.3 \\
\hline $3-5$ & $129(8.1)$ & 0.0 & 0.0 & 2.5 \\
\hline $6-9$ & $154(9.7)$ & 2.7 & 0.9 & 5.0 \\
\hline $10-19$ & $305(19.2)$ & 6.3 & 4.3 & 12.8 \\
\hline 20-39 & $368(23.1)$ & 22.5 & 21.0 & 20.6 \\
\hline 40 to more & $592(37.2)$ & 68.5 & 73.8 & 58.9 \\
\hline \multicolumn{5}{|l|}{$\begin{array}{l}\text { \# Times got drunk } \\
\text { in past year }\end{array}$} \\
\hline None & $86(5.9)$ & 0.9 & 0.5 & 1.4 \\
\hline $1-2$ & $319(21.9)$ & 3.6 & 3.8 & 7.7 \\
\hline $3-5$ & $258(17.7)$ & 8.0 & 8.8 & 15.7 \\
\hline $6-9$ & $188(12.9)$ & 7.1 & 11.9 & 13.1 \\
\hline $10-19$ & $208(14.3)$ & 14.3 & 17.3 & 17.7 \\
\hline $20-39$ & $193(13.2)$ & 21.4 & 24.2 & 17.1 \\
\hline 40 to more & $206(14.1)$ & 44.6 & 33.4 & 27.1 \\
\hline \multicolumn{5}{|l|}{$\begin{array}{l}\text { Self-description of } \\
\text { alcohol use }\end{array}$} \\
\hline Occasional drinker & $751(45.9)$ & 12.5 & - & 29.4 \\
\hline Light drinker & $307(18.8)$ & 5.4 & - & 11.5 \\
\hline Moderate drinker & $502(30.7)$ & 55.4 & - & 46.4 \\
\hline Heavy drinker & $75(4.6)$ & 26.8 & - & 12.6 \\
\hline
\end{tabular}

alcoholic, $68.5 \%$ of those who drank 40 or more times in the past year reported this fear. In contrast, only $58.9 \%$ of these frequent drinkers were flagged by the CAGE, even though a greater percentage of the total tested positive on the CAGE $(22.7 \%)$. Further, for both alternative criteria, no student who drank less than six times in the past year was flagged, but the CAGE identified $2.8 \%$ of these least frequent drinkers as probable abusers.

Using odds ratios as a measure of association, we compared the three indicators of problematic drinking to see which measure best identified students experiencing harmful consequences of alcohol use (see Table V). Of the 17 consequences for which three comparable odds ratios were available, the CAGE showed greater association in only one case ("someone said you should cut down"). A fear that one might be alcoholic was more highly associated with driving drunk, being arrested for driving drunk, having thoughts of suicide, being sexually harassed or harassing someone else-perhaps some of the most serious consequences-than either of the other indicators. Though the evidence is far from overwhelming, the single item question, "Have you been afraid you might be alcoholic?" may be the best identifier of at-risk students.

On the average, students flagged by the CAGE reported experiencing 6.9 of the 18 harmful outcomes in the past year, significantly higher than the average of 3.4 outcomes among those with negative CAGE results $(p<0.001)$. We considered whether the ability of the CAGE to recognize students at risk of harmful consequences varied depending on race/ethnicity or gender. We did not find that the odds ratios differed for gender or ethnicity/race in a statistically significant manner for any of the consequences. However, our results suggest that among women, the CAGE was more 
Table V. Comparison of Three Potential Indicators of Problematic Alcohol Use, and Their Relation to Negative Consequences of Alcohol Use

\begin{tabular}{lccc}
\hline \multicolumn{1}{c}{ Consequence } & Afraid alcoholic OR & Moderate/heavy drinker OR & CAGE OR \\
\hline Had hangover & 7.2 & $\mathbf{1 0 . 7}$ & 4.5 \\
Vomited & $\mathbf{4 . 8}$ & 4.7 & 4.6 \\
Felt embarrassed & $\mathbf{9 . 1}$ & 4.3 & 5.7 \\
Had memory loss & $\mathbf{6 . 7}$ & 6.2 & 4.8 \\
Missed class & 5.7 & $\mathbf{5 . 9}$ & 3.2 \\
Drove drunk & $\mathbf{3 . 8}$ & 3.5 & 2.4 \\
Got into an argument or fight & $\mathbf{4 . 6}$ & 4.6 & 3.7 \\
Were hurt or injured & 4.6 & $\mathbf{6 . 9}$ & 4.4 \\
Someone said to cut down & 11.2 & 4.6 & $\mathbf{1 2 . 2}$ \\
Performed poorly on a test & $\mathbf{7 . 0}$ & 4.5 & 4.6 \\
Were sexually harassed & $\mathbf{4 . 1}$ & 1.9 & 3.4 \\
Damaged property & 4.6 & $\mathbf{9 . 2}$ & 3.4 \\
Had trouble with police & 3.0 & $\mathbf{4 . 9}$ & 3.1 \\
Were afraid might be alcoholic & $\mathbf{6 . 1}$ & 9.8 & $\mathbf{1 7 . 1}$ \\
Friend threatened to leave & $\mathbf{5 . 5}$ & 3.8 & 4.2 \\
Sexually harassed someone & $\mathbf{9 . 5}$ & 4.9 & 3.0 \\
Thought about suicide & $\mathbf{1 3 . 9}$ & 3.1 & 7.2 \\
Were arrested for DUI & & 7.4 & 8.1 \\
\hline
\end{tabular}

Larger odds ratios (OR) indicate a stronger association with the consequence; largest OR for each consequence is in bold text.

highly associated with certain items but these trends were only marginally significant $(p<0.10)$. For both men and women, "were afraid you might be alcoholic" showed the greatest correlation with CAGE results.

While the CAGE may prove useful in identifying those suffering the harmful consequences of alcohol use, unfortunately, it cannot reassure us that those testing negative are not also suffering serious consequences from alcohol abuse. And despite the apparent sensitivity of the CAGE in identifying students who are at risk for these negative consequences, we draw two troubling conclusions from our results. First, a student who is not flagged or identified by the CAGE as a probable alcohol abuser still has a very good chance of experiencing several of the detrimental outcomes from alcohol abuse. For instance, over a quarter of those not testing positive on the CAGE reported having driven while intoxicated in the last 12 months. Eleven percent were hurt or injured as a result of drinking and $32 \%$ of students not identified by the CAGE had missed a class due to alcohol use. Among those not describing themselves as moderate or heavy drinkers, $24 \%$ had missed class, $20 \%$ had driven drunk, and $7 \%$ had been hurt or injured. Among those individuals who were not afraid of being alcoholic, $36 \%$ had missed class, $28 \%$ had driven drunk, and $15 \%$ had been hurt or injured. Notably, among students flagged as alcohol abusers on the CAGE, $61 \%$ missed classess, $45 \%$ drove drunk and $36 \%$ were hurt or injured but students; however, among students flagged by, "afraid you were an alcoholic," $76 \%$ missed classes, $59 \%$ drove drunk and $45 \%$ were hurt or injured. Second, the weakest association with the CAGE was with one of the most serious outcomes of drinkingdriving drunk $(\mathrm{OR}=2.4)$. These points lead us to seriously question the use of the CAGE as a screening instrument for problem drinking among this population. While screening instruments are often discussed in terms of their sensitivity, specificity, and positive and negative predictive values, a "true" or medical diagnosis must be known in order to calculate such measures. 
Our data revealed another concern regarding the ability of the CAGE to discriminate. We asked respondents to report the number of times they used alcohol (in the past year) and the number of times they had gotten drunk in the same period. We calculated the proportion of students in each response category who were flagged as problematic drinkers on the basis of the CAGE. While the percentage testing positive on the CAGE increased as the frequency of drinking increased, we did note some anomalies. Of those who reported using alcohol at least 40 times in the past year (592 students), 58.9\% were identified as probable alcohol abusers. However, of those who reported getting drunk at least 40 times (206 students), presumably a category containing a higher proportion of alcohol abusers, the percentage flagged by the CAGE was only $27.1 \%$. We believe that generally heavy alcohol users were less likely to recognize that they were drunk and probably got drunk less often despite more frequent alcohol use. In addition, some respondents may have answered the CAGE items with less accuracy than their lighter-drinking counterparts because of social desirability concerns. Our findings also suggest that heavy drinking behavior alone is not necessarily an indication of alcohol dependence or alcohol-related problems within the collegiate population.

We were interested in respondents' subjective assessment of their drinking. Respondents were asked whether they considered themselves occasional, light, moderate, or heavy drinkers (abstainers are excluded here); the CAGE was not correlated with this self-classification variable in any consistent manner. While the CAGE flagged $46.4 \%$ of moderate drinkers, only $12.6 \%$ of heavy drinkers were identified as probable alcohol abusers. Also, 29.4\% of occasional drinkers were positive on the CAGE, compared to only $11.5 \%$ of light drinkers. Again, these discrepancies suggest either that the CAGE works imperfectly in this population, or that self-described drinking behavior is not accurate.

Our data also suggest that the CAGE would be most useful if it was enhanced with other questions. Alternatives to the CAGE appeared more closely related to the number of times a student got drunk in the past 12 months. However, for both "afraid you might be alcoholic" and the "moderate/heavy drinker" indicators, we observed the anomalous trend we had seen with the CAGE. For both measures, the percentage of students being detected as problematic drinkers was higher for those reporting they used alcohol 40 or more times in the past year than for those reporting being drunk 40 or more times. Lastly, there was no meaningful association between "afraid you might be alcoholic" and self-described drinking behavior.

The fact that additional items may either enhance the CAGE or even function with greater specificity is supported by an earlier study by Heck. Heck (15) suggested that the CAGE may be ineffective in screening college students for alcohol problems because the CAGE detects only more severe patterns of problem drinking, and typically, college students have not been drinking long enough to develop these problems. In order to develop a more useful screening questionnaire, Heck suggested using the CAGE, plus additional items to assess quantity and frequency. He found several elements significantly discriminated between problem drinkers and normal drinkers: (1) endorsing the "cut down" and "annoyed" questions on the CAGE; (2) never or rarely choosing nonalcoholic beverages at social events; (3) driving under the influence at least six times in the past year and (4) having started regular alcohol use before college. Our conclusions closely resemble Heck's in that we also found five 
elements that appear associated with problem drinking. These include endorsing at least two items on the CAGE, being fearful about possible alcoholism, driving drunk within the past year and the quantity of alcohol consumed in the past year.

There are several limitations to our study. For instance, we utilized a crosssectional design with a sample drawn from one, large, Midwestern university; thus, generalizability is somewhat limited although we are encouraged that our data are similar to national data. Further, it is possible that fielding the survey during the end of the winter semester negatively affected our response rate, although by fielding it later in the semester, we were able to avoid the changes in drinking that often occur during the University's spring break. The survey took $20 \mathrm{~min}$ to complete and some students wrote to tell us that it was too long; we may have lost respondents who simply did not want to spend $30 \mathrm{~min}$ answering a survey. However, when considering the time element, we were forced to balance the length of the survey with our need for data. Finally, although the $68 \%$ response rate is adequate for a study of an undergraduate student population, nonresponse represented a limitation in the present study. In another web-based study conducted within the same student population, a telephone follow-up of nonrespondents to an e-mail invitation to participate in a Web survey revealed that the majority $(56.2 \%)$ of nonrespondents did not recall receiving the invitational e-mail (18). However, the study did not examine the substance-use behaviors of the nonrespondents and future college-based research would be well-served to examine the substance use behaviors of nonrespondents with follow-up studies.

\section{CONCLUSION}

Although binge drinking rates are not increasing among college students, they are also not decreasing $(19,20)$. In the United States this year, it is estimated that over 1000 college students will die from alcohol-related accidents and hundred thousands more will be either assaulted by students under alcohol's influence or suffer drinking-related injuries themselves $(21,22)$. Clearly, the negative consequences of heavy episodic drinking continue to take a toll on our campuses and we need to effectively identify the problem drinkers and then, intervene. In this paper, we argue that a modified version of the CAGE screening instrument, one of the most commonly used screening measures on college campuses is imperfect and a better assessment measure could be considered to identify problem drinkers within this population. While making any changes to the CAGE screening instrument would require a tremendous effort to educate/reeducate the clinical and medical professions, the authors believe the effort would be worth the benefit of creating a more developmentally sensitive screening instrument for college students.

\section{ACKNOWLEDGMENTS}

Funding for this project was provided by the University of Michigan, Ann Arbor, MI. The authors thank Market Strategies, Inc., Livonia, MI for assistance with data collection and two anonymous reviewers for their helpful comments on an earlier version of this manuscript. 


\section{REFERENCES}

1. Johnston LD, O'Malley PM, Bachman JG: Monitoring the Future National Survey Results on Drug Use, 1975-2000, Vol. II: College students and adults ages 19-40 (2001) NIH Publication No. 01-4925). Bethesda, MD: National Institute on Drug Abuse

2. Wechsler H, Lee JE, Kuo M, Lee H: College binge drinking in the 1990s: A continuing problem. J Am Coll Health 2000; 48(10):199-210

3. Aergeerts B, Buntinx F, Bande-Knops J, Vandermeulen C, Roelants M, Ansoms S, Fevery J: The value of CAGE, CUGE, AUDIT in screening for alcohol abuse and dependence among college freshman. Alcoholism Clin Exp Res 2000; 24:53-57

4. Heck EJ, Williams MD: Using the CAGE to screen for the drinking-related problems in college students. J Stud Alcohol 1995; 56:282-286

5. O'Hare T, Tran R: Predicting problem drinking in college students: Gender differences and the CAGE questionnaire. Addict Behav 1997; 22(1):13-21

6. Meryholtz L, Rosenberg H: Screening college students for alcohol problems: Psychometric assessment of the SASSI-2. J Stud Alcohol 1998; 439-446

7. Allen JP, Maisto S, Connors GJ: Self-report screening tests for alcohol problems in primary care. Arch Intern Med 1995; 155:1726-1730

8. Maisto S, Connors G, Allen J: Contrasting self-report screens for alcohol problems: A review. Alcoholism Clin Exp Res 1995; 19(6):1510-1516

9. Bisson J, Nadeau L, Demmers A: The validity and reliability of the CAGE scale to screen for heavy drinking and drinking problems in a general population survey. Addiction 1999; 94(5):715-722

10. Cherpitel C: Performance of screening instruments for identifying alcohol dependence in the general population, compared with clinical populations. Alcoholism Clin Exp Res 1998; 22(7):1399-1404

11. Cherpitel C: Screening for alcohol problems in the U.S. population: A comparison of the CAGE and TWEAK by gender, ethnicity, and services utilization. J Stud Alcohol 1999; 60:705-711

12. Presley CA, Meilman PW, Cashin JR, Lyerla R: Alcohol and Drugs on American College Campuses: Use, Consequences, and Perceptions of the Campus Environment, Vol. IV: 1992-94. Carbondale, IL: Southern Illinois University; 1996

13. Wechsler H, Davenport A, Dowdall G, Moykens B, Castillo S: Health and behavioral consequences of binge drinking in college: A national survey of students at 140 campuses. JAMA 1994; 272(21):1672-1677

14. McCabe SE: Gender differences in collegiate risk factors for heavy episodic drinking. J Stud on Alcohol 2002; 63(1):49-56

15. Heck EJ: Developing a screening questionnaire for problem drinking in college students. J Am Coll Health 1991; 39:227-231

16. Cashin JR, Presley, CA, Meilmen PW: Alcohol use in the greek system: Follow the leader? J Stud Alcohol 1998; 59:63-70

17. Wechsler H, Isaac N: Binge drinkers at Massachusetts Colleges: Prevalence, drinking style, time trends, and associated problems. JAMA 1992; 267:2929-2931

18. McCabe SE, Boyd CJ, Couper MP, Crawford S, d'Arcy H: Mode effects for collecting alcohol and other drug use data: Web and U.S. Mail. J Stud Alcohol 2002; 63:755-761

19. Wechsler H, Dowdall GW, Maenner, G Gledhill-Hoyt J, Lee H: Changes in binge drinking and related problems among American college students between 1993 and 1997. J Am Coll Health 1998; 47:57-68

20. Wechsler H, Lee JE, Kuo M, Lee H: Trends in College Binge Drinking During a Period of Increased Prevention Efforts: Findings from 4 Harvard School of Public Health College Alchol Study Surveys: 1993-2001. J Am Coll Health 2002; 50:201-217

21. Hitt E: Alcohol Abuse Takes Major Toll on College Students. Yahoo! Retrieved on November 02, 2001, from http://dailynews.yahoo.com/htx/nm/20011024/hl/alcohol_2.html (2001, October)

22. Hingson RW, Heeren T, Zakocs RC, Kopstein A, Wechsler H: Magnitude of alcohol-related mortality and morbidity among U.S. college students ages 18-24. J Stud Alcohol 2002; 63:136-144 